\title{
Intensified biochip system using chemiluminescence for the detection of Bacillus globigii spores
}

\author{
Dimitra N. Stratis-Cullum • Guy D. Griffin • \\ Joel Mobley • Tuan Vo-Dinh
}

Received: 30 November 2007 /Revised: 21 December 2007 / Accepted: 7 January 2008 / Published online: 27 January 2008

(C) Springer-Verlag 2008

\begin{abstract}
This paper reports the first intensified biochip system for chemiluminescence detection and the feasibility of using this system for the analysis of biological warfare agents is demonstrated. An enzyme-linked immunosorbent assay targeting Bacillus globigii spores, a surrogate species for Bacillus anthracis, using a chemiluminescent alkaline phosphatase substrate is combined with a compact intensified biochip detection system. The enzymatic amplification was found to be an attractive method for detection of low spore concentrations when combined with the intensified biochip device. This system was capable of detecting approximately $1 \times 10^{5}$ Bacillus globigii spores. Moreover, the chemiluminescence method, combined with the selfcontained biochip design, allows for a simple, compact system that does not require laser excitation and is readily adaptable to field use.
\end{abstract}

\footnotetext{
D. N. Stratis-Cullum ( $₫)$

US Army Research Laboratory, AMSRD-ARL-SE-EO, 2800 Powder Mill Road,

Adelphi, MD 20783, USA

e-mail: dimitra.stratiscullum1@us.army.mil

G. D. Griffin · T. Vo-Dinh $(\bowtie)$

Fitzpatrick Institute for Photonics,

Departments of Biomedical Engineering and Chemistry,

Duke University,

2589 Fitzpatrick Center,

Durham, NC 27708, USA

e-mail: tuan.vodinh@duke.edu

J. Mobley

Department of Physics and Astronomy,

The University of Mississippi,

1025 NCPA,

Oxford, MS 38677, USA
}

Keywords Biochips · Biosensors immunoassays · ELISA · Spectroscopy B Bacillus globigii . Chemiluminescence . Spores $\cdot$ Intensifier $\cdot$ Bioluminescence

\section{Introduction}

Over the last decade, there has been a growing interest in the rapid detection of pathogens for homeland defense [1-3]. Of particular concern among the potential biological warfare (BW) agent candidates for use as a weapon is Bacillus anthracis for a number of reasons. For example, $B$. anthracis can be produced and released in large numbers in the spore form, which is highly resistant to inactivation. Moreover, it is a highly pathogenic organism, requiring medical attention within $24-48 \mathrm{~h}$ of initial exposure, and infective doses have been estimated to be very low (e.g., 8,000 to 10,000 spores inhaled) [3]. Consequently, there is a definite need for practical detection devices capable of identification and quantitation of $\mathrm{BW}$ agents, such as $B$. anthracis spores.

One of the primary approaches for detection and speciesspecific identification of BW agents is based on immunological recognition [2, 4-8]. In addition to being both highly sensitive and selective, immunological techniques can also be easily adapted to field use [2,9-11]. Unlike nucleic acidbased analyses, immunological methods do not require a cell/spore lysis step for the extraction of DNA or RNA, as surface antigens can be targeted. This is particularly advantageous for the detection of bacterial spores, such as $B$. anthracis, as it is very difficult to disrupt the strong, resistant shell of the spore form [12].

In the development process of portable detection devices, the tradeoff between the size of the instrumentation and detection sensitivity is an important consideration. However, 
when detecting biological agents (e.g., B. anthracis) in the field, sensitivity is essential. One route to sensitive detection is to utilize a method that incorporates amplification of the signal, such as enzyme-linked immunosorbent assays (ELISA) [4, 6, 13]. In an ELISA, an antibody specific to the target species is immobilized onto a solid support or platform. As illustrated in Fig. 1, the antigen (i.e., target species) binds selectively to the capture antibody, and is thus immobilized on the platform. For these studies, the target of interest is Bacillus globigii spores, a surrogate species for $B$. anthracis. Following immobilization, a labeled second antibody, which is used for detection, specifically recognizes another epitope on the spore surface. Signal amplification is made possible by modifying the detector antibody(ies) to include a conjugated enzyme. The final amplification step of the assay involves addition of a substrate upon which the enzyme (conjugated to the detector antibody) functions with a very high turnover rate to produce a detectable product. This measurable endpoint, typically the production of a colored reaction product, is usually detected spectrophotometrically and the absorbance of the reaction product is used to quantify the amount of antigens or target species present in the sample. By using a substrate that produces either a fluorogenic or chemiluminescent product (upon enzymatic cleavage by the conjugated enzyme) the sensitivity of the assay can be enhanced even further $[6,14]$.

Development of a method incorporating a chemiluminescent product allows for a number of potential advantages over fluorescent methods including extreme sensitivity, as well as simpler instrumentation [5, 15-18]. More specifically, with chemiluminescence, photons are generated only when the reactants are present, unlike fluorescence where an excitation source could induce nonspecific radiation from either scattering or background excitation of the sample matrix. Since problems of light scattering, nonselective excitation, and source instability are absent, chemiluminescence possesses an inherently low background, allowing for a very sensitive analysis. Moreover, since an excitation source and associated optics required for fluorescence analyses are eliminated, detection can be achieved with a simpler system that is readily adaptable to field use. Previously, we have developed an integrated circuit biochip that has demonstrated great potential for field use [6]. This biochip device has a number of distinct advantages over alternate biosensing technologies including a fabrication process based on complementary metal oxide semiconductor (CMOS) technology and multianalyte detection $[6,13$, 19-21]. For example, the CMOS fabrication process allows for application-specific circuitry (i.e., signal amplification and filtering) to be integrated into the chip, thereby significantly reducing the size and power requirements of the system. Another important consideration is that the CMOS process is very cost-effective, which is ideal for mass producing portable detection devices. Furthermore, the chip is composed of an array of individual detector elements, each of which could be devoted to the detection of a different

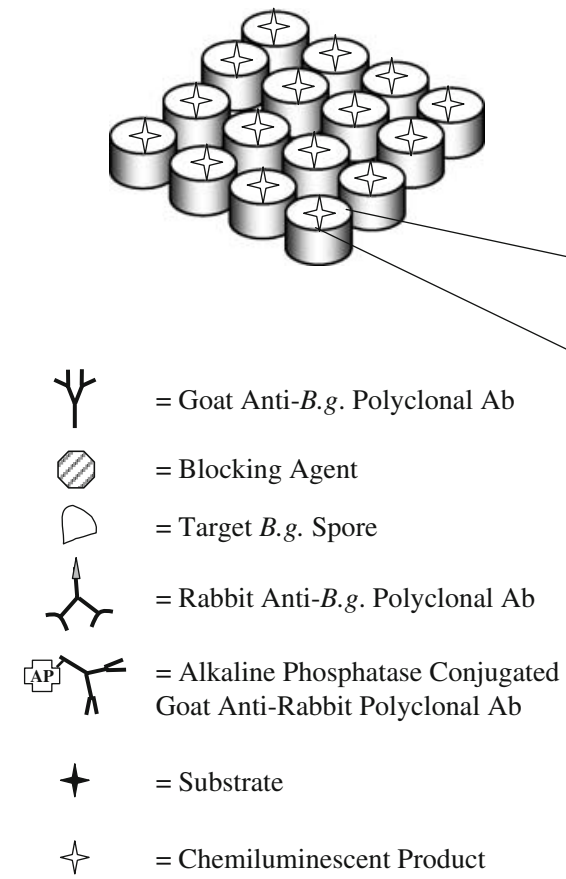

Fig. 1 Schematic diagram of the enzyme-linked immunosorbent assay, used for the detection of Bacillus globigii spores. The final stage of the bioassay involves enzymatic cleavage of the substrate by the enzyme-conjugated detector antibody, converting the substrate into the chemiluminescent product 
biological agent for multiplexed detection. For example, in this work a $4 \times 4$ photosensor array was used, which could be capable of performing 16 simultaneous biowarfare agent analyses in a single, compact unit.

In the current work, the first biochip device with an integrated intensifier enabling chemiluminescence detection is reported and the feasibility of using this system for the detection of Bacillus globigii spores is demonstrated. This system has a number of potential advantages over previous designs, including elimination of a laser and associated optics used for sample excitation in fluorescence systems. Furthermore, this simpler design, along with the self-contained biochip integrated circuit, could allow for the development of a small yet sensitive system for field use.

\section{Experimental}

Intensified biochip detection system

The miniature biochip detection system, consisting of intensifier and integrated circuit photosensing array chip, is illustrated schematically in Fig. 2. The resulting chemiluminescence from each sample chamber was incident on a modified night vision scope intensifier (Moonlight NV-100 009415 San Diego, CA). In this way, an image of the

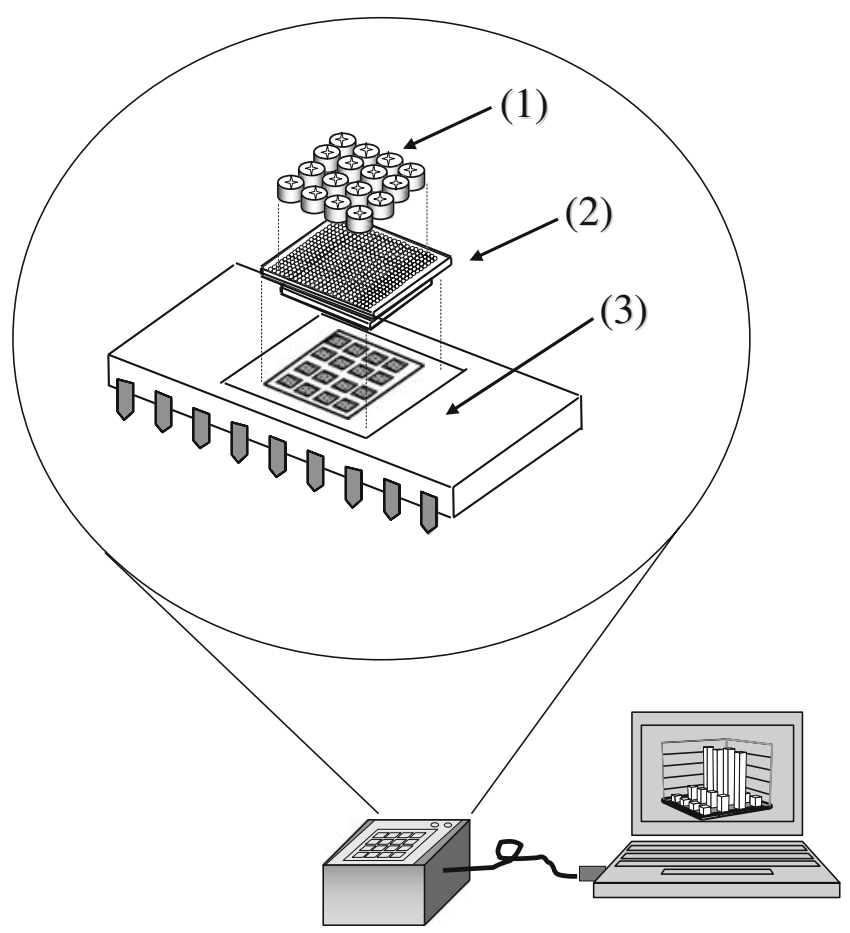

Fig. 2 Schematic diagram of the miniature biochip detection system, consisting of 1 sample chambers containing the chemiluminescent assay, 2 intensifier for signal amplification, and 3 integrated circuit photosensing array chemiluminescence light originating from the 16 individual sample chambers can be amplified prior to detection by each one of the 16 elements of the photosensing chip. Four amplifier gain settings, which were available by varying the voltage applied to the intensifier, are referred to in the text as settings $1-4$.

The integrated circuit biochip prototype was developed previously by the Vo-Dinh group at Oak Ridge National Laboratory [20]. Each photosensor is $900-\mu \mathrm{m}$ square in size and is arranged in a $1-\mathrm{mm}$ grid array. Through a $1.2-\mu \mathrm{m} \mathrm{n}$ well CMOS fabrication process, application-specific circuitry was integrated into the chip for digital control of signal filtering and amplification. The resulting output voltage from each of the individual photosensors was recorded using a laptop computer with in-house written Labview software. The chemiluminescence signal intensity, originating from the 16 locations, was then correlated to the concentration of target B. globigii spores.

\section{ELISA procedure for detection of $B$. globigii spores}

An enzyme-linked immunosorbent assay (ELISA) for antibody-based capture and identification of Bacillus globigii (B.g.) spores was used in conjunction with the biochip detection instrumentation. As illustrated in Fig. 1, antibodies specific to a surface antigen on the B.g. spore (goat anti-B. globigii diluted to $10 \mu \mathrm{g} \mathrm{mL}^{-1}$ in $0.1 \mathrm{M}$ carbonate buffer, $\mathrm{pH}$ 9.6) were immobilized onto a Nunc maxisorp protein binding platform (Nunc Maxisorp 96-well plate surface) overnight at $4{ }^{\circ} \mathrm{C}$. For all other antibody binding steps, incubation times were approximately $1 \mathrm{~h}$ in order to insure complete binding. However, these times could be reduced significantly for field use $[6,14]$.

After immobilization of the capture antibodies, the remaining binding sites were blocked for $1 \mathrm{~h}$ at room temperature using a bovine serum albumin (BSA) diluent/ blocking solution concentrate, diluted 1:10 in distilled water (Kirkegard and Perry Laboratories (KPL), Gaithersburg, MD). Following blocking, the immobilized antibodies were then incubated with Bacillus globigii spores (Var. Niger, Baker Labs Dugway Proving Grounds, UT) diluted to various concentrations in phosphate-buffered saline (PBS) at $37{ }^{\circ} \mathrm{C}$ from a solution in the same buffer. Bacillus globigii stock solution concentration was determined by both serial dilutions and standard plate counts grown on a generic growth medium (trypticase soy agar) as well as through counting using a hemocytometer. Following incubation with the spores, the wells were washed thoroughly in PBS $+0.5 \%$ Tween 20 to remove any unbound target species. Subsequently, a detector antibody (rabbit anti- $B$. globigii), recognizing another epitope on the B.g. spore surface, was diluted in BSA diluent/blocking solution concentrate (1:15 dilution in distilled water) to a final 


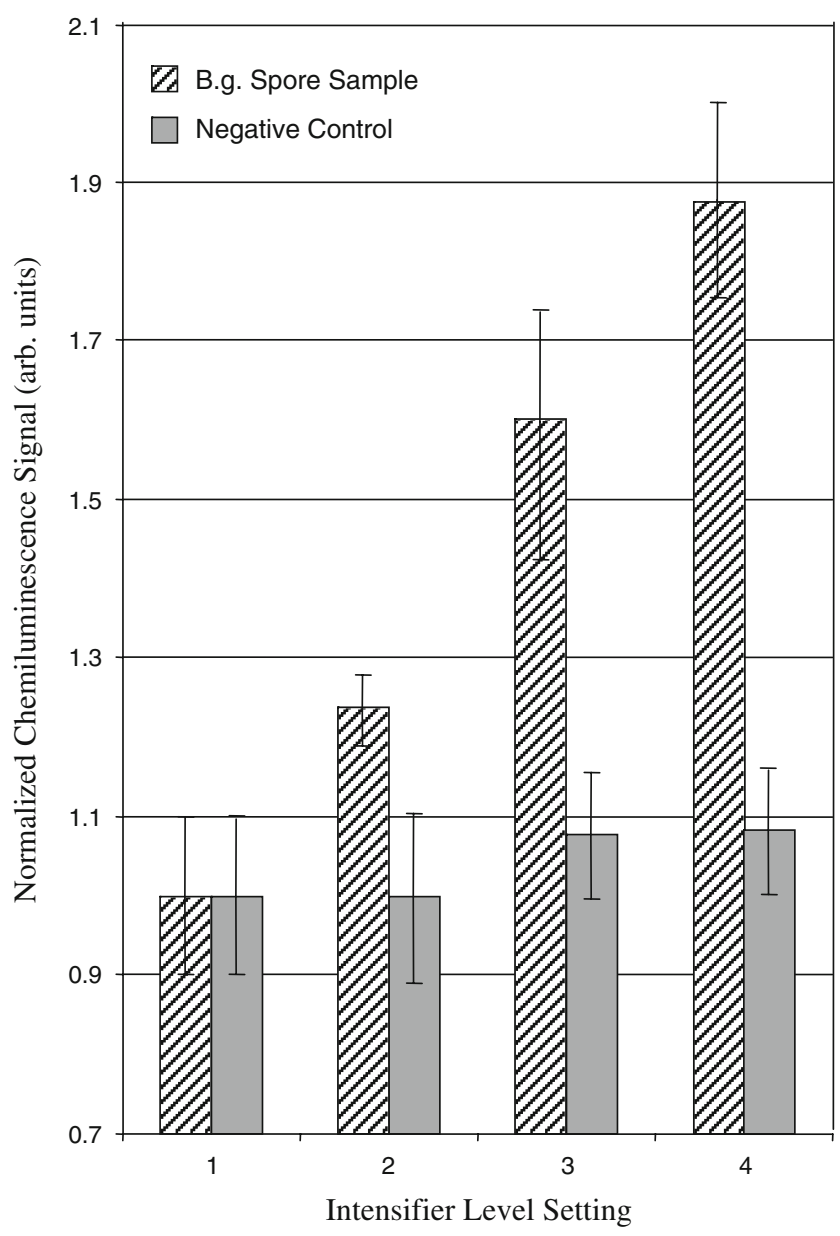

Fig. 3 Amplification of chemiluminescence signal from ELISA product using the intensified biochip system levels $1-4$ for a Bacillus globigii spore sample $\left(5.6 \times 10^{6}\right.$ spores) and a negative control ( 0 spores)

concentration of $5 \mu \mathrm{g} \mathrm{mL} L^{-1}$ and incubated at $37^{\circ} \mathrm{C}$ with the captured spores before being washed several times (PBS + $0.5 \%$ Tween 20). The final antibody, goat anti-rabbit IgG $(\mathrm{H}+\mathrm{L})$ conjugated with alkaline phosphatase (Jackson Immunoresearch Laboratories, Avondale, PA), was diluted 1:3,000 in alkaline phosphatase (AP) stabilizer (KPL, Gaithersburg, MD), and was incubated at $37{ }^{\circ} \mathrm{C}$ with the sample complex. The unbound enzyme-antibody conjugate was then removed through several washes as described above. Finally, $100 \mu \mathrm{L}$ of the chemiluminescent substrate based on a novel 1,2-dioxetane (Intergen Bold APS 540) was incubated with the immunocomplex for $1 \mathrm{~h}$ to yield a detectable chemiluminescent product emitting 540-nm light. For all experiments here, the reactions were carried out under "saturated substrate" conditions. The results from three separate bioassays were recorded ten times each per chamber and averaged for each of the data points described below. The error bars shown on the graphical results represent plus or minus one standard deviation calculated from these replicate assay experiments and measurements.

\section{Results and discussion}

One of the greatest challenges to biowarfare detection is the development of field-deployable instrumentation capable of performing sensitive analyses. Not surprisingly, current research efforts of many groups aim to improve detection capabilities by incorporating some form of amplification of either the target (e.g., polymerase chain reaction for DNA targets) or indirect amplification of the signal (e.g., enzymelinked immunosorbent assay for protein targets). Another method of achieving signal amplification is by enhancing the capabilities of the detection device by using an intensified system. However, with a typical fluorescence-based analysis, background photons originating from nonspecific

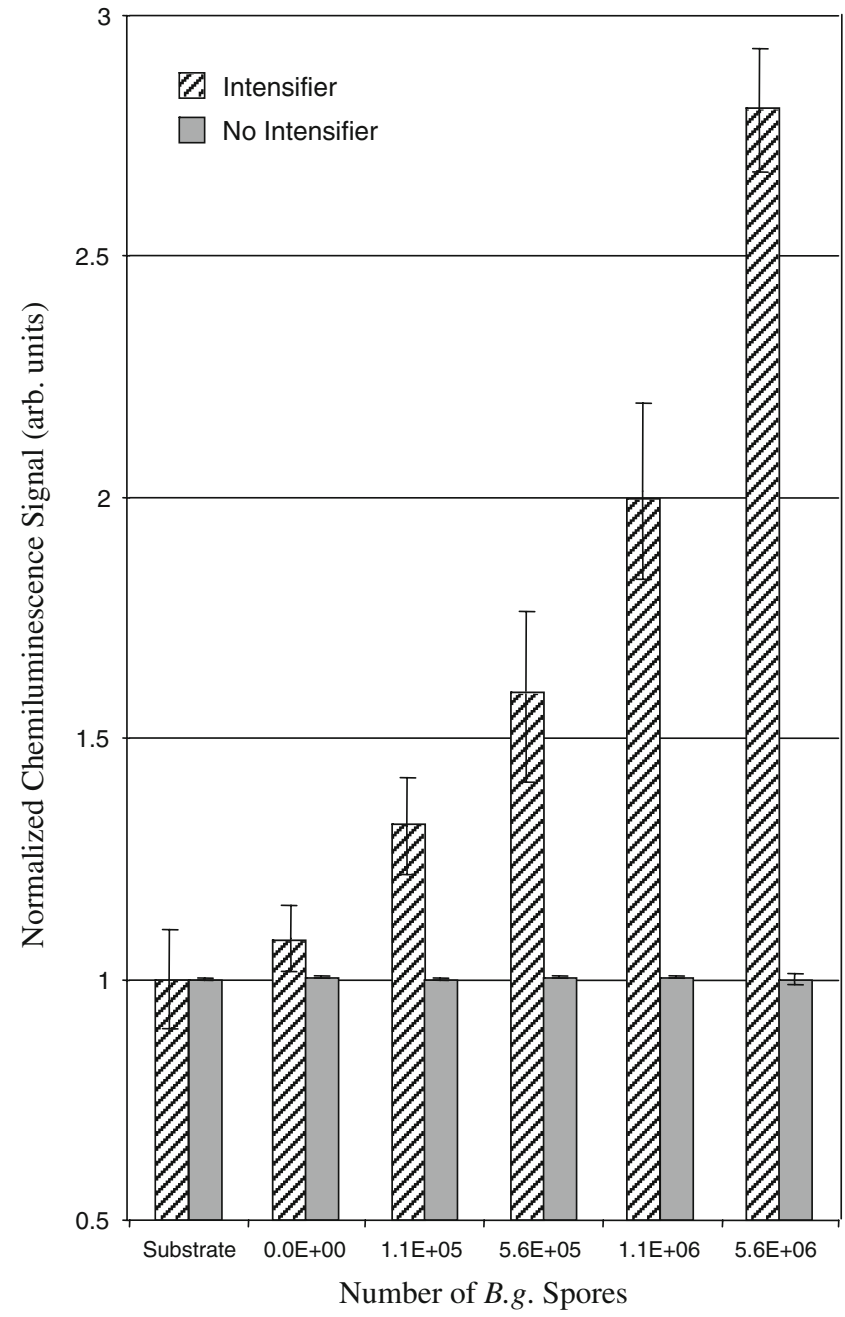

Fig. 4 Detection of Bacillus globigii spores using the ELISA method and with an intensified biochip system and nonintensified biochip system. The chemiluminescence intensity of the enzymatic product is shown for different Bacillus globigii spore concentrations. The negative control $(0.0 \mathrm{E}+00)$, was obtained using identical conditions, except there was no introduction of the spore samples. Also shown is the signal from the substrate alone, which has not been converted to the chemiluminescent product 
excitation and emission or light scattering of the light source will also be amplified by the intensifier.

Intensified biochip system for chemiluminescence detection

To investigate the feasibility of incorporating an intensifier with the portable biochip system for the analysis of a chemiluminescence reaction product, the signal as a function of four intensifier levels, (produced by increasing voltage on the microchannel plate) for a positive sample and a negative control were investigated, as shown in Fig. 3. For both the positive (ca. $5.6 \times 10^{6}$ B.g. spores) and the negative control sample (0 spores) shown, an ELISA producing a chemiluminescence reaction product was coupled to the intensified biochip system. Increases in signal were observed for the positive B.g. sample when using increasing amplification levels of the microchannel plate. The negative control signal represents the level of nonspecific binding, as all assay steps were conducted with the exception that there was no introduction of the B.g. spore target. Since chemiluminescent photons are generated only when the reactants are present, very little signal is observed for the negative control sample, thereby providing a low background. Upon comparison of the positive control signal gain with increasing intensifier settings to the negative control background at the same intensifier settings, it is clear that the highest intensifier setting produced the greatest signal to background advantage and was hence used throughout the rest of these studies. The highest intensifier setting did not appear to cause saturation at the signal levels investigated.

Detection of Bacillus globigii spores using the intensified biochip system

Once the optimal intensifier level was determined, the feasibility of using the compact intensified biochip system for the detection of anthrax spores was investigated using a range of known concentrations of B.g. spores. These results are summarized in Fig. 4 which shows the intensity of the chemiluminescent product of the enzymatic reaction, obtained after incubation with the substrate, for the different B.g. spore concentrations. For comparison, the negative control, using all assay steps except the introduction of B.g. spores is shown. The background intensity of the unreacted substrate control, which has not been converted to the product, is also shown. In general, when using the intensified biochip, the chemiluminescence intensity was found to increase with increasing spore concentrations. Also shown are the results obtained for the same B.g. spore sample measurements taken without the intensifier. Comparison of the two sets of data indicated that the intensified biochip system allowed for a sensitive analysis that was not achievable using the nonintensified system.
This method produced little background levels, as evidenced by the comparable signal intensity of the negative control relative to that observed for the substrate. One possible source of this low background includes nonspecific interactions of either the rabbit anti-B.g. or alkaline phosphatase-conjugated goat anti-rabbit detector antibodies used in the immunoassay as described in Fig. 1. Consequently, it is possible that the low background levels and error bars produced in these studies could be further reduced through systematic optimization of the blocking agent, reagent concentrations, and washing conditions, for minimal nonspecific interactions and maximum assay reproducibility.

These results show that enzyme-based amplification offers a potentially attractive alternative to nucleic acid-based amplification methods (e.g., polymerase chain reaction), since a sensitive analysis can be performed without requiring a cell-lysing step that is particularly difficult to achieve with the hard, resistant shells of spores. In addition, the ELISA method utilizing a chemiluminescent product is characterized by an extremely low background, which couples well to the compact intensified biochip.

\section{Conclusion}

This is the first report of an intensified biochip system for the detection of biological agents. Through the combination of the sensitive intensified biochip system ideal for low-background chemiluminescence measurements, and the enzyme-based signal amplification, detection of $5 \times 10^{5}$ spores was demonstrated. Advantages of this system include elimination of a laser and associated optics used for sample excitation in fluorescence systems, and a chemiluminescence-based method characterized by an inherently low background, which is ideally suited for an intensified device. Furthermore, the simple design, along with the self-contained biochip integrated circuit, could allow for the development of a small yet sensitive system for field use. Moreover, multiple biowarfare agents could be detected within a single, compact unit, as each biosensing element could be devoted to the detection of a different biological agent.

Acknowledgments This research was sponsored by the U.S. Department of Energy, Chemical and Biological National Security Program, under contract DE-AC05-00OR22725 with UT-Battelle, LLC. The authors also thank Dr. Arpad Vaas (Oak Ridge National Laboratory) for his help in the preparation of the Bacillus samples used in this work.

\section{References}

1. Inglesby TV, O'Toole T, Henderson DA, Bartlett JG, Ascher MS et al (2002) JAMA 287:2236-2252

2. Shriver-Lake LC, Ligler FS (2005) IEEE Sens J 5:751-756 
3. Walt DR (2000) Anal Chem 72:738A-746A

4. Gehring AG, Irwin PL, Reed SA, Tu SI (2006) J Rapid Methods Autom Microbiol 14:349-361

5. Rongen HAH, Hoetelmans RMW, Bult A, Vanbennekom WP (1994) J Pharm Biomed Anal 12:433-462

6. Stratis-Cullum DN, Griffin GD, Mobley J, Vass AA, Vo-Dinh T (2003) Anal Chem 75:275-280

7. Iqbal SS, Mayo MW, Bruno JG, Bronk BV, Batt CA, Chambers JP (2000) Biosens Bioelectron 15:549-578

8. Griffin GD, Williams MW, Stratis-Cullum DN, Vo-Dinh T (2003) Amplification technologies for optical detection. In: Vo-Dinh $\mathrm{T}$ (ed) Biomedical photonics handbook, Chap 55. CRC, Boca Raton, pp 51-71

9. Anderson GP, King KD, Gaffney KL, Johnson LH (2000) Biosens Bioelectron 14:771-777

10. King KD, Vanniere JM, Leblanc JL, Bullock KE, Anderson GP (2000) Environ Sci Technol 34:2845-2850
11. Ligler FS, Anderson GP, Davidson PT, Foch RJ, Ives JT et al (1998) Environ Sci Technol 32:2461-2466

12. Belgrader P, Okuzumi M, Pourahmadi F, Borkholder DA, Northrup MA (2000) Biosens Bioelectron 14:849-852

13. Vodinh T, Griffin GD, Ambrose KR (1986) Appl Spectrosc 40:696-700

14. Yacoub-George E, Meixner L, Scheithauer W, Koppi A, Drost S et al (2002) Anal Chim Acta 457:3-12

15. Cheek BJ, Steel AB, Torres MP, Yu YY, Yang HJ (2001) Anal Chem 73:5777-5783

16. Cheek BJ, Steel AB, Yang HJ (2001) Biophys J 80:164A

17. Luo LR, Zhang ZJ, Hou LY (2007) Anal Chim Acta 584:106-111

18. Marquette CA, Blum LJ (2006) Anal Bioanal Chem 385:546-554

19. Stokes DL, Griffin GD, Tuan VD (2001) Fresenius J Anal Chem 369:295-301

20. Vo-Dinh T, Alarie JP, Isola N, Landis D, Wintenberg AL, Ericson MN (1999) Anal Chem 71:358-363

21. Vo-Dinh T, Cullum B (2000) Fresenius J Anal Chem 366:540-551 